\title{
UCLA/FNPL Underdense Plasma Lens Experiment: Results and Analysis
}

\author{
M.C. Thompson, ${ }^{* \dagger}$, H. Badakov ${ }^{\dagger}$, J.B. Rosenzweig ${ }^{\dagger}$, G. Travish ${ }^{\dagger}$, R. \\ Fliller**, G. M. Kazakevich**, P. Piot'**, J. Santucci** ${ }^{* *}$. Li ${ }^{\ddagger}$ and R. \\ Tikhoplav ${ }^{\ddagger}, \uparrow$ \\ * Lawrence Livermore National Laboratory, Livermore, California, 90095 \\ UCLA Department of Physics and Astronomy, Los Angeles, California, 90095 \\ ** Fermi National Accelerator Laboratory, Batavia, Illinois, 60510 \\ ${ }^{\ddagger}$ University of Rochester, Rochester, New York, 14627
}

\begin{abstract}
Focusing of a $15 \mathrm{MeV}, 16 \mathrm{nC}$ electron bunch by a gaussian underdense plasma lens operated just beyond the threshold of the underdense condition has been demonstrated. The strong $1.9 \mathrm{~cm}$ focal length plasma lens focused both transverse directions simultaneously and reduced the minimum area of the beam spot by a factor of 23. Analysis of the beam envelope evolution observed near the beam waist shows that the spherical aberrations of this underdense lens are lower than those of an overdense plasma lens, as predicted by theory. Time resolved measurements of the focused electron bunch are also reported and compared to simulations.
\end{abstract}

Keywords: plasma lens, wakefield accelerator, final focus, beam optics

PACS: $52.40 . \mathrm{Mj}, 41.75 . \mathrm{Ht}, 29.27 . \mathrm{Eg}, 52.35 . \mathrm{Mw}$

\section{INTRODUCTION}

Plasma lenses are of great interest to the relativistic beam physics community because they can provide radially symmetric focusing gradients equivalent to a quadrupole lens gradient of the order $1 \mathrm{MT} / \mathrm{m}$, which exceeds the strength of conventional devices by many orders of magnitude $[1,2]$. Additionally, it has been shown theoretically that adiabatic plasma lenses [3] can overcome the synchrotron radiation-induced limit on final focus spot size [4]. Consequently, there is great interest in using plasma lenses to achieve the small spot sizes and high luminosity necessary at the interaction point of future $e^{+} e^{-}$colliders such as the proposed International Linear Collider (ILC). We recently made the first observation of plasma lens focusing of a relativistic electron beam in the low-aberration underdense-regime: $n_{b} \gtrsim n_{p} / 2$, where $n_{b}$ and $n_{p}$ are the beam and plasma densities, respectively.

Plasma lenses operate in either the overdense $\left(n_{b}<<n_{p}\right)$ or underdense regime. In the overdense case the plasma electrons spatially configure so that the plasma ions cancel the beam space charge and thus allow the beam to focus under its magnetic self-forces. These self-forces are not linear in distance from the beam axis $(r)$, or uniform in distance along the beam axis $(z)$, which leads to significant aberrations in the overdense case [2]. In the underdense case the strong electric field created by the space charge of the electron beam ejects the plasma electrons from the beam region entirely, leaving a uniform ion column. It can be shown that the radial-focusing electric-force of this ion column is 


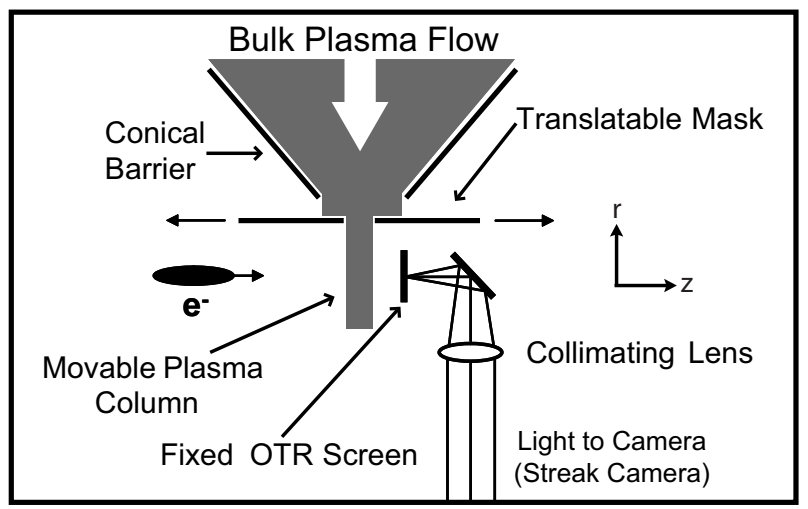

FIGURE 1. Schematic of the plasma lens apparatus.

linear and given by $F_{\text {radial }}=-2 \pi n_{p} e^{2} r$, where $e$ is the electron charge. An underdense plasma lens is, therefore, theoretically free of spherical aberration in the limit that the ion column is as wide as the beam and the ions are immobile. The head of the beam is not focused in an underdense plasma lens because of the finite response time of the plasma electron density distribution.

Focusing by overdense plasma lenses of up to $4 \mathrm{MT} / \mathrm{m}$ strength has been demonstrated in previous experiments [5 - 9]. Focusing in the low-aberration underdense-plasma-lens regime, however, has not been previously observed. The related concept of underdense ion channel formation was validated in experiments designed to examine beam matching and transport in a long plasma channel $[10,11]$, but this channeling work did not address underdense lens focusing. Our observations show that spherical aberrations of our underdense lens are well below the lower bound on overdense plasma lens aberrations. This is true even at the threshold of underdense operation, where $n_{b} \approx n_{p} / 2$. This threshold was initially noted in simulations by $\mathrm{Su}$ et al. [2] and is somewhat surprising in that, from an examination of only electrostatic forces, one would suppose $n_{b} \approx n_{p}$ should be the threshold. The physical basis for an underdense threshold at $n_{b} \approx$ $n_{p} / 2$ has not been discussed previously. We have made an experimental investigation of the threshold regime and are developing a model for the threshold case.

\section{EXPERIMENTAL METHODOLOGY}

In the experiment a $14.8 \mathrm{MeV}$ electron beam is focused by a plasma lens of approximately gaussian profile in $z$ with FWHM of $19.3 \mathrm{~mm}$ and peak density $n_{p, \text { peak }}=4.9 \mathrm{x}$ $10^{12} \mathrm{~cm}^{-3}$. This plasma lens is uniform in $r$ on the length scale of the beam, has an average focusing strength $K=2561 \mathrm{~m}^{-2}$ over an effective length of $l=20.5 \mathrm{~mm}$, and a focal length $(f=1 / K l)$ of $1.9 \mathrm{~cm}$. In magnetic quadrupole units, the lens has an average focusing gradient of $126 \mathrm{~T} / \mathrm{m}$, which is about 40 times stronger than the magnetic qudrupole fields used to transport the beam to our experiment.

The plasma lens was created using a direct current electrical discharge transversely offset from the beam orbit as illustrated in Fig. 1. A bulk plasma column of approximate width $5 \mathrm{~cm}$ FWHM is produced using our pulsed argon discharge plasma source [12] 
and allowed to diffuse toward the beam path under the weak confinement of a $53 \mathrm{G}$ solenoidal magnetic field aligned with the plasma flow. A conical stainless steel barrier suppresses diffusion into the interaction area and a thin slice of the static plasma column is selected using a translatable mask with a $1.25 \mathrm{~cm}$ wide slit. This arrangement allows the distance between the plasma lens column and the fixed optical transition radiation (OTR) diagnostic screen to be varied without altering the OTR collection optics. An invacuum, $160 \mathrm{~mm}$ focal length, 2 inch diameter lens provides quasi-parallel collimation of the OTR produced when the electron beam emerges from the back of a polished aluminum foil. The OTR is then transported either $30 \mathrm{~cm}$ to a conventional CCD camera or $7 \mathrm{~m}$ to a streak camera where the light is imaged onto the streak slit using a single $280 \mathrm{~mm}$ focal length lens.

The electron beam for the experiment is provided by the Fermilab NICADD Photoinjector Laboratory (FNPL) facility [13]. The FNPL injector is a $15 \mathrm{MeV}$ electron source consisting of a normal conducting L-band RF gun with a $\mathrm{Cs}_{2} \mathrm{Te}$ photo-cathode and a 9-cell superconducting post-accelerator cavity. After acceleration, the electron beam is propagated at a tight focus through a $10 \mu \mathrm{m}$ thick aluminum vacuum-isolation foil and refocused into the plasma lens experiment. Scattering in the aluminum foil significantly increases the beam emittance. The beam is transported $2.2 \mathrm{~m}$ from the vacuum foil to the center of the plasma lens.

\section{UNDERDENSE PLASMA FOCUSING}

The effect of linear transverse focusing elements, whether plasma or magnetic, on an electron beam traveling in the $z$ direction can be modeled (neglecting space charge effects) by the beam envelope equation:

$$
\frac{d^{2} \sigma_{x}}{d z^{2}}+K \sigma_{x}=\frac{\varepsilon_{x}^{2}}{\sigma_{x}^{3}}
$$

where $\sigma_{x}$ is the rms beam size in $x, \varepsilon_{x}$ is the geometric beam emittance in $x$, and $K$ is the focusing strength of the lens. The equivalent equation for the other transverse direction

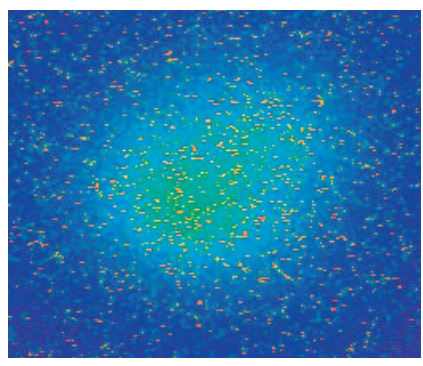

Plasma Off - 5 electron

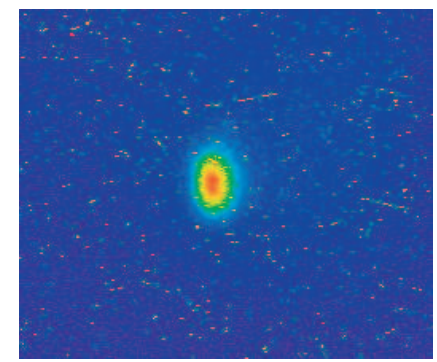

Plasma On - 1 electron

FIGURE 2. Images of the unfocused (left) and focused (right) electron beam OTR displayed with the same scaled-to-intensity color map. To overcome the limited dynamic range of the camera, the unfocused image (left) is the integration of 5 beam pulses. 

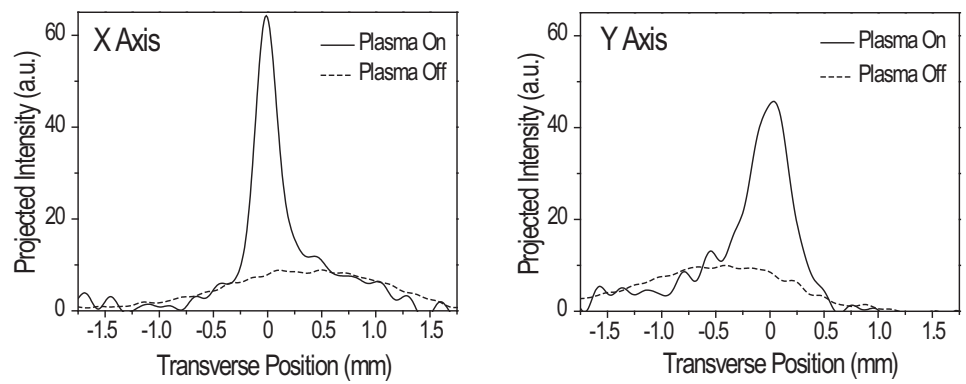

FIGURE 3. Plots of the projected intensity of the beam images shown in Fig. 2 (normalized to 1 electron pulse) in the $\mathrm{x}$ axis (left) and $\mathrm{y}$ axis (right).

can be written by substituting $y$ for $x$. For an underdense plasma lens $K=2 \pi r_{e} n_{p} / \gamma$, where $r_{e}$ is the classical electron radius, and $\gamma$ is the Lorentz factor [14]. Detailed predictions of the plasma lens focusing of the beam core can be made by solving Eq. 1 using a function $n_{p}(z)$ that describes the shape of our thick plasma lens. The envelope equation can also be use to describe the focusing of lenses with aberrations. A general way to do this is to define an effective emittance $\varepsilon_{\text {eff }}=\sqrt{\varepsilon_{0}^{2}+\beta_{0} \varepsilon_{0} \delta \theta^{2}}$ for use in Eq. 1 which includes both the original emittance of the beam $\varepsilon_{0}$ and the extra angular spread resulting from the lens aberrations $\delta \theta$ [15]. The aberration induced angular spread can be defined as $\delta \theta=\left(\sqrt{\beta_{0} \varepsilon_{0}} / f\right)(\Delta K / K)$ where $\beta_{0}=\left(\sigma_{0}^{2} / \varepsilon_{0}\right)$ is the beam beta-function at the lens entrance, $f$ is the lens focal length, and $\Delta K$ is the rms amount the lens strength deviates from linear expectation over the beam. Su et al. have shown that the minimum spherical aberration of an overdense plasma lens focusing a gaussian beam is $\Delta K / K=0.21$ [2]. As stated previously, an underdense plasma lens can theoretically be spherical aberration free $(\Delta K / K=0)$ in the ideal limit.

In order to understand the spherical aberrations of our plasma lens we made a series of round beam focusing measurements with different spacings between the plasma lens and OTR screen. These experiments used a $14.8 \mathrm{MeV}$ electron beam with initial dimensions $x_{\mathrm{FWHM}}=1629 \pm 126 \mu \mathrm{m}, y_{\mathrm{FWHM}}=1544 \pm 120 \mu \mathrm{m}, \mathrm{Q}=16.2 \pm 1.7 \mathrm{nC}$, and $\sigma_{t}=22 \pm 3$ ps. Consequently the initial peak beam density was $n_{b, p e a k}=2.2 \pm 0.4 \times 10^{12} \mathrm{~cm}^{-3}$. Uncertainties originate from shot to shot variation of the beam. Peak density of the plasma lens is $n_{p, \text { peak }}=4.9 \times 10^{12} \mathrm{~cm}^{-3}$ so $n_{b, p e a k} \approx n_{p, p e a k} / 2$ putting the experiment just on the boundary of the underdense regime, in terms of beam and plasma peak densities, at the entrance to the plasma lens. This is a lower bound however since the beam is substantially denser compared to the edge of the gaussian plasma column which it first encounters and, since the lens is thick, the envelope equation and simulations show the beam core has compressed somewhat by the time it reaches the center of the plasma lens. We can, therefore, unequivocally state that the beam focusing is governed by underdense dynamics at the threshold of the regime. When optimally focused, a beam waist with $x_{\mathrm{FWHM}}=259 \pm 22 \mu \mathrm{m}$ and $y_{\mathrm{FWHM}}=423 \pm 41 \mu \mathrm{m}$ is achieved as shown in Fig. 2 and Fig. 3. These measurements reflect the size of the focused beam core as our camera lacked sufficient dynamic range to simultaneously record unfocused portions of the beam (halo) along with the focused core. The demagnification factor of 6.29 in $x$ and 

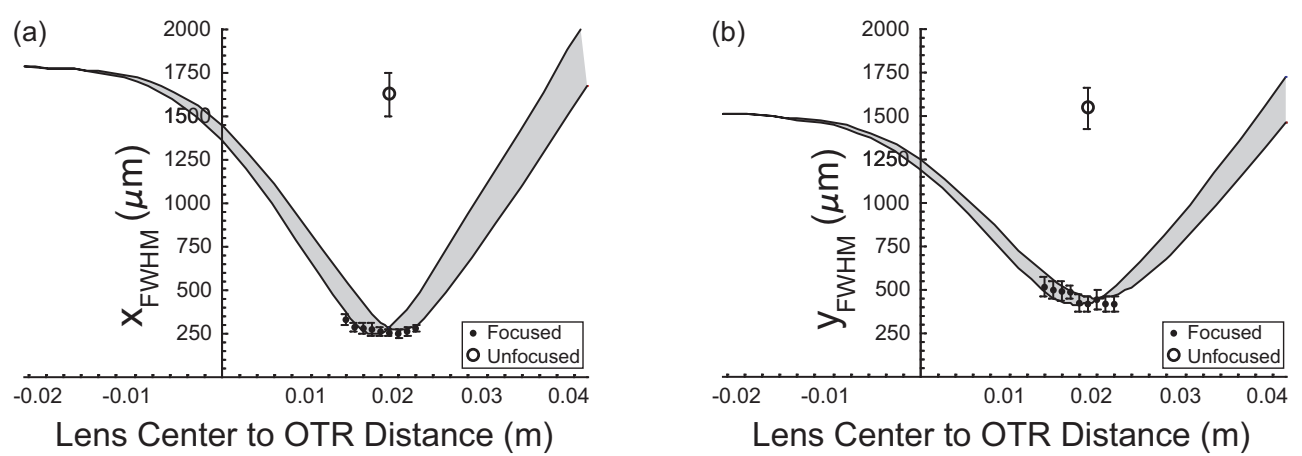

FIGURE 4. Measurements of underdense plasma lens focusing in the $x$ axis (a) and $y$ axis (b) of a round beam shown with solutions to the envelope equation (solid lines and shaded regions). The shaded regions indicate the shift in focal length caused by the variation in plasma lens parameters.

3.65 in $y$ means that the transverse area of the focused beam is reduced by a factor of 23 .

Fitting the predictions of the envelope equation to the focusing data obtained in the round beam case, Fig. 4 reveals a great deal about the plasma lens and its aberrations. The unfocused beam envelope is calculated using the initial conditions at the vacuum foil. The calculated values match the observed unfocused beam sizes at the OTR foil well and the beam sizes and convergence at the entrance to the plasma lens are taken from this solution. Note that the manner in which we generated our plasma lens leads to slight variation in parameters. In this case, the plasma lens was measured to have peak density that varied from $4.56-5 \times 10^{12} \mathrm{~cm}^{-3}$ over the range of the data points. The width of the lens was constant at $19.3 \pm 1.9 \mathrm{~mm}$ over the range of interest. The variation in lens parameters leads to a slight variation in the lens focal length and therefore a broadening of the observed depth of focus as shown in Fig. 4. While the focal length is determined by the known plasma lens parameters, the size of the beam waist is determined by the parameter $\varepsilon_{e f f}$ which was obtained by a best fit to the data. For the curves shown in Fig. $4 \varepsilon_{e f f, x, n}=110 \mathrm{~mm}$-mrad and $\varepsilon_{e f f, y, n}=155 \mathrm{~mm}$-mrad, where we have followed convention and used normalized emittance $\varepsilon_{n}=\beta \gamma \varepsilon$. A value of $\varepsilon_{x, n}=87 \mathrm{~mm}$-mrad was measured by a quadrupole scan downstream of the vacuum isolation foil. Using the above expression for $\varepsilon_{e f f}$ we can immediately calculate $\Delta K_{x} / K_{x}=0.076 \pm 0.006$ which is well below the overdense lens minimum of 0.21 and thus strongly indicative of underdense operation.

We were not instrumented to make an independent measurement of $\varepsilon_{y, n}$, which makes the analysis of aberrations in this axis more uncertain. If we assume similar lens performance in $y$ as in $x\left(\Delta K_{x} / K_{x}=\Delta K_{y} / K_{y}=0.076 \pm 0.006\right)$ it implies $\varepsilon_{y, n}=147 \mathrm{~mm}$-mrad. Such an emittance asymmetry is quite likely considering that $\varepsilon_{x, n}$ and $\varepsilon_{y, n}$ were dominated by scattering in the vacuum isolation foil and that the beam spot symmetry could not be guaranteed during this interaction. The magnitude of $\varepsilon_{y, n}$ implied is also plausible since measured values of $\varepsilon_{x, n}$ ranged from 87 - $165 \mathrm{~mm}$-mrad over several days of the experiment. We therefore conclude that the $y$ axis data are also consistent with underdense operation.

The observed aberration of our plasma lens has a chromatic component. The small 

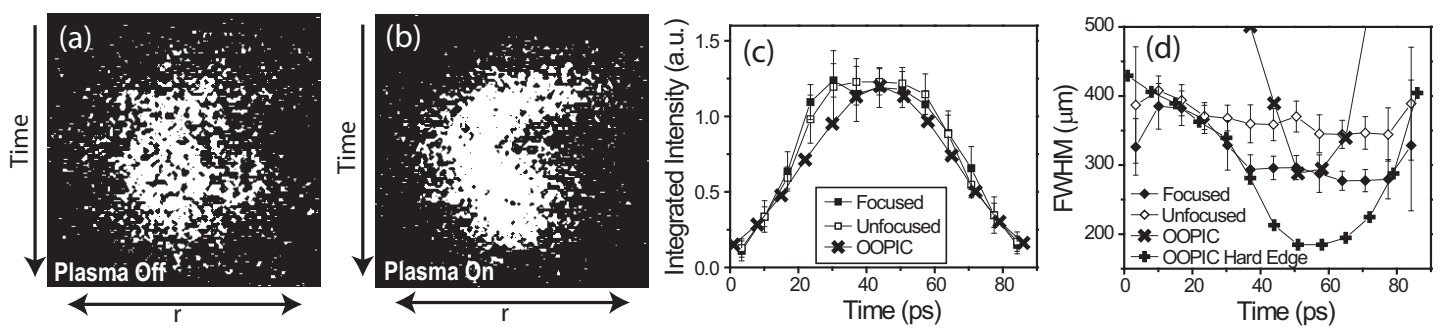

FIGURE 5. Representative streak camera images of the unfocused (a) and plasma focused beam (b). Time slice dependence of the beam intensity (c) and transverse FWHM (d) for the unfocused beam, the plasma focused beam, and the results of simulations.

energy spread of the electron beam was generated primarily by longitudinal plasma wake fields. Experimental limitations lead us to operate in an accelerator-like mode with $k_{p, p e a k} \sigma_{z}=2.75$, rather than in the preferred plasma lens limit $k_{p} \sigma_{z} \gg 1$, where $k_{p}^{-1}=c / \omega_{p}$ is the plasma skin depth. Simulations of the beam / plasma interaction indicate an induced beam energy spread of $2.5 \%$ full width. From the total energy spread it is straightforward to calculate $(\Delta K / K)_{\text {chromatic }} \leq 0.025$, indicating that the observed aberrations are predominately spherical.

A series of time resolved measurements of the plasma focusing were made by imaging the beam derived OTR onto the slit of a 2 ps resolution streak camera. An example of the images recorded on the streak camera, along with an analysis of many shots, is shown in Fig. 5. As expected, the intensity profile of the focused beam in the time domain remains roughly gaussian while the beam is radially larger at the head than in the middle or tail. Measurements were made by examining 6.7 ps wide slices of the beam. While the time domain behavior of the plasma focusing is in general agreement with theoretical predictions, and the focused beam diameter observed on the steak images matches the value measured using the OTR screen CCD camera, there is clearly clipping of the signal in the transverse direction. The effect of this clipping is illustrated by the two traces derived from the same simulation with the particle-in-cell code OOPIC [16] shown in Fig. 5(d). The lower OOPIC trace was drawn using only particles within a set radius to simulate the effect of a hard aperture while the upper trace contains the full distribution. To our knowledge there were no limiting apertures to explain this effect. The clipping may be the result of losses over the long light transport to the streak camera that reduced the low intensity edges of the beam below the detection threshold.

\section{CONCLUSIONS}

In conclusion, we have measured extreme demagnification of a high brightness electron beam by a strong underdense plasma lens and shown that, as predicted, this lens has lower aberrations than overdense lenses even near the boundary of the underdense regime $\left(n_{b} \gtrsim n_{p} / 2\right)$. We conjecture that the lens has a reasonably well formed ion column in this threshold regime due to the additive effect of the electrostatic forces and the mutual repulsion between the beam and plasma return currents. We are exploring the validity of this explanation through simulation. Operation at the boundary of the 

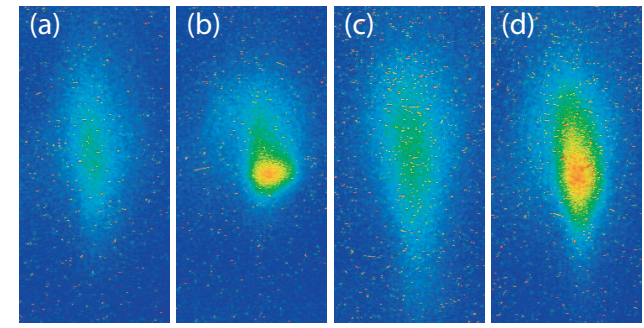

FIGURE 6. Images of unfocused $(a, c)$ and plasma focused $(b, d)$ asymmetric electron beams of initial transverse aspect ratio $\mathrm{x}: \mathrm{y}=1: 2.38(\mathrm{a}, \mathrm{b})$ and $\mathrm{x}: \mathrm{y}=1: 2.7(\mathrm{c}, \mathrm{d})$. All four OTR images are displayed with the same scaled to intensity color map.

underdense regime may be an attractive plasma lens scenario since it combines low aberration with minimal beam density. Lowering $n_{b}$ may be one way to mitigate the problem of ion motion [17] for ILC class beams.

Further investigation of our results, along with more detailed simulations, should improve our understanding the dynamics of plasma focusing in the underdense boundary regime. Expanding the analysis to include our observations of clear near-threshold underdense plasma lens focusing of modestly asymmetric beams (Fig. 6) may also be instructive. These asymmetric beam measurement are a first step toward the flat beam underdense lens experiments that are needed to validate the concept for ILC.

\section{ACKNOWLEDGMENTS}

This work was performed under the auspices of the US Department of Energy under Contract No. DE-FG03-92ER40693 and W-7405-ENG-48.

\section{REFERENCES}

1. P. Chen, Part. Accel. 20, 171-182 (1987).

2. J. J. Su, T. Katsouleas, J. M. Dawson, and R. Fedele, Phys. Rev. A 41, 3321 (1990).

3. P. Chen, K. Oide, A. M. Sessler, and S. S. Yu, Phys. Rev. Lett. 64, 1231 (1990).

4. K. Oide, Phys. Rev. Lett. 61, 1713-1715 (1988).

5. H. Nakanishi, et al., Phys. Rev. Lett. 66, 1870 (1991).

6. G. Hairapetian, et al., Phys. Rev. Lett. 72, 2403 (1994).

7. R. Govil, W. P. Leemans, E. Y. Backhaus, and J. S. Wurtele, Phys. Rev. Lett. 83, 3202 (1999).

8. P. R. Bolton for the E150 Collaboration, Int. J. Mod. Phys. A 18, 2843 (2003).

9. J. S. T. Ng, et al., Phys. Rev. Lett. 87, 244801 (2001).

10. N. Barov, M. E. Conde, W. Gai, and J. B. Rosenzweig, Phys. Rev. Lett. 80, 81 (1998).

11. C. E. Clayton, et al., Phys. Rev. Lett. 88, 154801 (2002).

12. M. C. Thompson, J. B. Rosenzweig, and G. Travish, Rev. Sci. Instrum. 76, 013303 (2005).

13. J.-P. Carneiro, et al., "First Results of the Fermilab High-Brightness RF Photoinjector," in Proc. PAC 1999, IEEE, 1999, p. 2027.

14. N. Barov, and J. B. Rosenzweig, Phys. Rev. E 49, 4407 (1994).

15. J. B. Rosenzweig, B. Cole, D. J. Larson, and D. B. Cline, Part. Accel. 24, 11 (1988).

16. D. L. Bruhwiler, et al., Phys. Rev. ST Accel. Beams 4, 101302 (2001).

17. J. B. Rosenzweig, A. M. Cook, A. Scott, M. C. Thompson, and R. B. Yoder, Phys. Rev. Lett. 95, 195002 (2005). 